\title{
ITERATIVE SOLUTIONS OF WIENER-HOPF INTEGRAL EQUATIONS*
}

\author{
$\mathrm{By}$ \\ TAI TE WU AND TAI TSUN WU \\ Harvard University, Cambridge, Massachusetts
}

\begin{abstract}
A method of iteration is used to study integral equations of the WienerHopf type. In the case of a single integral equation, it is found that the iterative solution can be summed to give the known results. In the case of two coupled integral equations, where the general solution is not known, the iterative solution can be reduced to expressions in closed form, in the sense of a finite number of quadratures, only in some very special cases. Two such special cases are discussed in detail.
\end{abstract}

1. Introduction. It is well-known that the linear integral equation

$$
f(\xi)-\int_{0}^{\infty} k(\xi-\eta) f(\eta) d \eta=g(\xi)
$$

for $\xi>0$, can be solved for $f(\xi)$ by the method of Wiener and Hopf [1]. If the Fourier transform of $f(\xi)$ is defined by $f^{+}(x)=\int_{0}^{\infty} f(\xi) e^{i x \xi} d \xi$ and $f^{-}(x)=\int_{-\infty}^{0} f(\xi) e^{i x \xi} d \xi$, which can be analytically continued into the entire upper and lower half-planes respectively, (1a) transforms to

$$
f^{+}(x)+f^{-}(x)-K(x) f^{+}(x)=g^{+}(x),
$$

where $K(x)=\int_{-\infty}^{\infty} k(\xi) e^{i x \xi} d \xi$ and $g^{+}(x)=\int_{0}^{\infty} g(\xi) e^{i x \xi} d \xi$. It is convenient to use the Plemelj formulae, $f^{+}(x)+f^{-}(x)=\phi(x)$ and $f^{+}(x)-f^{-}(x)=(\pi i)^{-1} \int_{-\infty}^{\infty}[\phi(y) /(y-x)] d y$ where the principal value should be taken for the integral. Then (1b) is equivalent to a singular integral equation of the form

$$
\phi(x)+\frac{\alpha(x)}{\pi i} \int_{-\infty}^{\infty} \frac{\phi(y)}{y-x} d y=h(x),
$$

where

$$
\alpha(x)=-K(x) /[2-K(x)] \text { and } h(x)=2 g^{+}(x) /[2-K(x)] .
$$

A solution of (2) is given by [2]

$$
\begin{gathered}
\phi(x)=h(x)\left[1-\alpha^{2}(x)\right]^{-1}-\frac{1}{\pi i} \alpha(x)\left[1-\alpha^{2}(x)\right]^{-1 / 2} \exp \left[-\frac{1}{2 \pi i} \int_{-\infty}^{\infty} \frac{d y}{y-x} \log \frac{1+\alpha(y)}{1-\alpha(y)}\right] \\
\cdot \int_{-\infty}^{\infty} \frac{h(v) d v}{v-x}\left[1-\alpha^{2}(v)\right]^{-1 / 2} \exp \left[\frac{1}{2 \pi i} \int_{-\infty}^{\infty} \frac{d z}{z-v} \log \frac{1+\alpha(z)}{1-\alpha(z)}\right] .
\end{gathered}
$$

It is convenient to use the notation of Hilbert transforms

$$
(\mathcal{F C} \phi)(x)=\frac{1}{\pi i} \int_{-\infty}^{\infty} \frac{\phi(y) d y}{y-x} .
$$

If $\phi$ is square integrable, then $\phi=\mathcal{C}^{2} \phi$, at least in the sense of limit in the mean [3].

*Received June 18, 1962. 
With this notation, (2) can be written as $\phi+\alpha \mathcal{Y} \phi=h$. If $\alpha$ is sufficiently "small", then this can be solved by iteration with the result

$$
\phi=h-\alpha \mathfrak{H C h}+\alpha \mathfrak{H} \alpha \mathcal{H} h-\cdots
$$

In Sec. 2, the relation between the well-known solution (3) and the iterative solution (5) is studied. More precisely, a method is found whereby (5) can be summed to yield (3). This procedure of summation is only formal in many cases because its justification seems to require that the maximum value of $\alpha$ be less than 1 .

So far as a single equation of the Wiener-Hopf type is concerned, the complete solution is given by (3), and hence the method of summing the iterative solution (5) yields nothing new. In the case of coupled Wiener-Hopf integral equations, solutions are not known except in those cases where reduction to a single equation is possible. In Sec. 3, a modification of the method used in Sec. 2 is applied to a system of two coupled Wiener-Hopf equations. As expected, the general case cannot be solved by this procedure. However, in several special cases, where the elements of the matrix $\alpha$ satisfy certain specific assumptions, the coupled integral equations can be solved in closed forms, in the sense that the solutions can be written in terms of a finite number of integrals of known function. Unfortunately, the conditions to be fulfilled by the matrix $\alpha$ are quite complicated, and no physical problem has yet been found wherein these conditions are fulfilled, and at the same time, reduction to a single integral equation is not trivial. The relation of the iteration procedure to the usual Wiener-Hopf "splitting" procedure is briefly discussed in Sec. 4 .

2. Iterative solution of a single equation. Equation (5) is going to be studied by changing the orders of integration. The basic formula to be used is that of Poincare and Bertrand [4]:

$$
\frac{1}{(\pi i)^{2}} \int_{-\infty}^{\infty} \frac{d y}{y-x} \int_{-\infty}^{\infty} \frac{f(y, z) d z}{z-y}=f(x, x)+\frac{1}{(\pi i)^{2}} \int_{-\infty}^{\infty} d z \int_{-\infty}^{\infty} \frac{f(y, z) d y}{(y-x)(z-y)}
$$

Using a partial fractions development of the last term, it can be shown that for $f(y, z)=\alpha_{1}(y) \alpha_{2}(z),(6)$ is

$$
\begin{aligned}
& \frac{1}{(\pi i)^{2}} \int_{-\infty}^{\infty} \frac{\alpha_{1}(y) d y}{y-x} \int_{-\infty}^{\infty} \frac{\alpha_{2}(z) d z}{z-y}=\alpha_{1}(x) \alpha_{2}(x) \\
& \quad+\frac{1}{(\pi i)^{2}} \int_{-\infty}^{\infty} \frac{\alpha_{2}(z) d z}{z-x} \cdot \int_{-\infty}^{\infty} \frac{\alpha_{1}(y) d y}{y-x}-\frac{1}{(\pi i)^{2}} \int_{-\infty}^{\infty} \frac{\alpha_{2}(z) d z}{z-x} \int_{-\infty}^{\infty} \frac{\alpha_{1}(y) d y}{y-z},
\end{aligned}
$$

which, by the notation of (4), may be written alternatively as

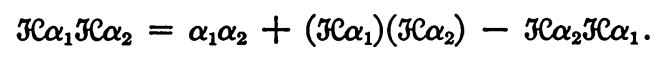

Repeated applications of (7) give

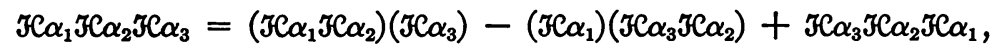

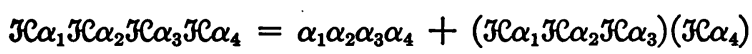

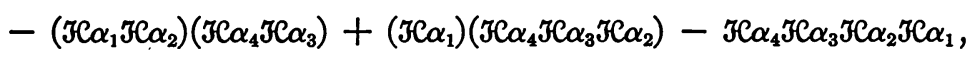

etc. Note that there is an extra term when the number of $\alpha$ 's is even. The substitution of this system of equalities into (5) then gives, after some rearrangement

$$
\phi=\left(1-\alpha^{2}\right)^{-1} h-\alpha A \Im C B h,
$$


where

$$
\begin{aligned}
& A=1-F C \alpha+F C \alpha \Im \alpha-\cdots, \\
& B=1+F C \alpha+F C \alpha C \alpha+\cdots .
\end{aligned}
$$

Thus the solution of (2) is reduced to the determination of $A$ and $B$ (each independent of $h$, as expected). A comparison of (10) with (3) gives

$$
\begin{aligned}
& A=\left(1-\alpha^{2}\right)^{-1 / 2} \exp \left(-\frac{1}{2} \mathfrak{H} \log \frac{1+\alpha}{1-\alpha}\right), \\
& B=\left(1-\alpha^{2}\right)^{-1 / 2} \exp \left(\frac{1}{2} \mathfrak{H} \log \frac{1+\alpha}{1-\alpha}\right) .
\end{aligned}
$$

These equalities can also be obtained directly from (11), as shown in Appendix A.

3. Iterative solution of coupled equations. The above procedure is next applied to a system of two coupled singular integral equations which, in matrix form, is given by

where

$$
\phi+\alpha \Re \phi=h,
$$

$$
\phi=\left[\begin{array}{l}
\phi_{1} \\
\phi_{2}
\end{array}\right], \quad h=\left[\begin{array}{l}
h_{1} \\
h_{2}
\end{array}\right], \quad \text { and } \quad \alpha=\left[\begin{array}{ll}
\alpha_{11} & \alpha_{12} \\
\alpha_{21} & \alpha_{22}
\end{array}\right] .
$$

The iterative solution of (13) is also given by (5). Equation (10) still holds provided that the definition of $B$ is generalized to

$$
B=\left[1+\Im C H \alpha^{T}+\Im C \alpha^{T} \Im C \alpha^{T}+\cdots\right]^{T},
$$

where the superscript $T$ denotes the transpose.

It is convenient to use the following notations:

$$
\begin{aligned}
& \theta_{1}=\left(1-\alpha_{11}^{2}\right)^{-1 / 2} \exp \left(-\frac{1}{2} \mathcal{H C} \log \frac{1+\alpha_{11}}{1-\alpha_{11}}\right), \\
& \theta_{2}=\left(1-\alpha_{22}^{2}\right)^{-1 / 2} \exp \left(-\frac{1}{2} \mathcal{H C} \log \frac{1+\alpha_{22}}{1-\alpha_{22}}\right), \\
& \theta_{3}=\left(1-\alpha_{11}^{2}\right)^{-1 / 2} \exp \left(\frac{1}{2} \mathcal{H C} \log \frac{1+\alpha_{11}}{1-\alpha_{11}}\right), \\
& \theta_{4}=\left(1-\alpha_{22}^{2}\right)^{-1 / 2} \exp \left(\frac{1}{2} \mathcal{H C} \log \frac{1+\alpha_{22}}{1-\alpha_{22}}\right),
\end{aligned}
$$

which are generalizations of (12). Note that $\theta_{1} \theta_{3}=\left(1-\alpha_{11}^{2}\right)^{-1}$ and $\theta_{2} \theta_{4}=\left(1-\alpha_{22}^{2}\right)^{-1}$.

Under the additional assumption that

$$
\begin{aligned}
\alpha_{21}= & \theta_{1}^{-1} \theta_{4}^{-1}\left(\alpha_{12} \theta_{2} \theta_{3}-\frac{1}{2} \mathcal{H}\left[\alpha_{11}-\alpha_{22}\right] \alpha_{12} \theta_{2} \theta_{3}\right)\left\{\mu^{2}-\frac{1}{4} \alpha_{12}^{2} \theta_{2}^{2} \theta_{3}^{2}\left(\alpha_{11}-\alpha_{22}\right)^{2}\right. \\
& \left.+\frac{1}{2} \alpha_{12} \theta_{2} \theta_{3}\left(1-\alpha_{11} \alpha_{22}\right) \mathcal{C}\left[\alpha_{11}-\alpha_{22}\right] \alpha_{12} \theta_{2} \theta_{3}-\frac{1}{4}\left(\mathcal{H C}\left[\alpha_{11}-\alpha_{22}\right] \alpha_{12} \theta_{2} \theta_{3}\right)^{2}\right\}^{-1},
\end{aligned}
$$

where $\mu$ is any constant, the matrices $A$ and $B$ are given by

$$
A=\left[\begin{array}{cc}
\theta_{1} P & -\theta_{1} Q \\
\alpha_{22} \alpha_{21}\left(1-\alpha_{22}^{2}\right)^{-1} \theta_{1} P-\theta_{2} \Im \mathcal{C} \alpha_{21} \theta_{1} \theta_{4} P & \theta_{2}-\alpha_{22} \alpha_{21}\left(1-\alpha_{22}^{2}\right)^{-1} \theta_{1} Q+\theta_{2} \Im \mathcal{H} \alpha_{21} \theta_{1} \theta_{4} Q
\end{array}\right],
$$


and

$$
B=\left[\begin{array}{cc}
\theta_{3}+\alpha_{11} \alpha_{21}\left(1-\alpha_{11}^{2}\right)^{-1} \theta_{4} R+\theta_{3} \mathcal{H} \alpha_{21} \theta_{1} \theta_{4} R & \theta_{4} R \\
\alpha_{11} \alpha_{21}\left(1-\alpha_{11}^{2}\right)^{-1} \theta_{4} S+\theta_{3} \mathcal{H} \alpha_{21} \theta_{1} \theta_{4} S & \theta_{4} S
\end{array}\right],
$$

where

$$
\begin{aligned}
& \boldsymbol{P}=\frac{1}{2}\left(1-C_{0}\right)^{-1}\left\{\frac{\mu}{\mu-C_{1}}\left[1-C_{2}^{2}\left(\mu-C_{1}\right)^{-2}\right]^{-1 / 2} \exp \left(\frac{1}{2} \mathcal{H C} \log \frac{\mu-C_{1}+C_{2}}{\mu-C_{1}-C_{2}}\right)\right. \\
& \left.+\frac{\mu}{\mu+C_{1}}\left[1-C_{2}^{2}\left(\mu+C_{1}\right)^{-2}\right]^{-1 / 2} \exp \left(-\frac{1}{2} \mathfrak{H C} \log \frac{\mu+C_{1}+C_{2}}{\mu+C_{1}-C_{2}}\right)\right\}, \\
& Q=\frac{1}{2}\left(1-C_{0}\right)^{-1}\left\{\frac { \mu } { \mu - C _ { 1 } } [ 1 - C _ { 2 } ^ { 2 } ( \mu - C _ { 1 } ) ^ { - 2 } ] ^ { - 1 / 2 } \left[-\alpha_{11} \alpha_{12} \theta_{2} \theta_{3}+3 C \alpha_{12} \theta_{2} \theta_{3}\right.\right. \\
& -\exp \left(-\frac{1}{2} \mathcal{H C} \log \frac{\mu-C_{1}+C_{2}}{\mu-C_{1}-C_{2}}\right) \mathfrak{H C} \frac{C_{2}}{\mu-C_{1}}\left(-\alpha_{11} \alpha_{12} \theta_{2} \theta_{3}+\mathcal{H C} \alpha_{12} \theta_{2} \theta_{3}\right) \\
& \left.\cdot\left[1-C_{2}^{2}\left(\mu-C_{1}\right)^{-2}\right]^{-1 / 2} \exp \left(\frac{1}{2} \nVdash \log \frac{\mu-C_{1}+C_{2}}{\mu-C_{1}-C_{2}}\right)\right] \\
& +\frac{\mu}{\mu+C_{1}}\left[1-C_{2}^{2}\left(\mu+C_{1}\right)^{-2}\right]^{-1 / 2}\left[-\alpha_{11} \alpha_{12} \theta_{2} \theta_{3}+\Im C \alpha_{12} \theta_{2} \theta_{3}\right. \\
& +\exp \left(\frac{1}{2} \mathfrak{H C} \log \frac{\mu+C_{1}+C_{2}}{\mu+C_{1}-C_{2}}\right) \mathcal{H C} \frac{C_{2}}{\mu+C_{1}}\left(-\alpha_{11} \alpha_{12} \theta_{2} \theta_{3}+\mathfrak{F C}_{12} \theta_{2} \theta_{3}\right) \\
& \left.\left.\cdot\left[1-C_{2}^{2}\left(\mu+C_{1}\right)^{-2}\right]^{-1 / 2} \exp \left(-\frac{1}{2} \mathfrak{H} \log \frac{\mu+C_{1}+C_{2}}{\mu+C_{1}-C_{2}}\right)\right]\right\} \text {, } \\
& R=\frac{1}{2}\left(1-C_{0}\right)^{-1}\left\{\frac { \mu } { \mu - C _ { 1 } } [ 1 - C _ { 2 } ^ { 2 } ( \mu - C _ { 1 } ) ^ { - 2 } ] ^ { - 1 / 2 } \left[\alpha_{22} \alpha_{12} \theta_{2} \theta_{3}+\Im C \alpha_{12} \theta_{2} \theta_{3}\right.\right.
\end{aligned}
$$

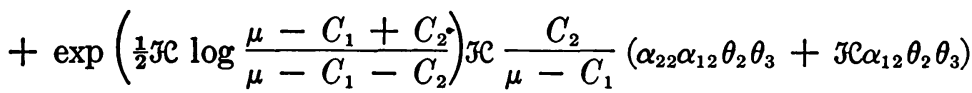

$$
\begin{aligned}
& \left.\cdot\left[1-C_{2}^{2}\left(\mu-C_{1}\right)^{-2}\right]^{-1 / 2} \exp \left(-\frac{1}{2} \mathfrak{H} \log \frac{\mu-C_{1}+C_{2}}{\mu-C_{1}-C_{2}}\right)\right] \\
& +\frac{\mu}{\mu+C_{1}}\left[1-C_{2}^{2}\left(\mu+C_{1}\right)^{-2}\right]^{-1 / 2}\left[\alpha_{22} \alpha_{12} \theta_{2} \theta_{3}+\mathcal{H} C \alpha_{12} \theta_{2} \theta_{3}\right. \\
& -\exp \left(-\frac{1}{2} \mathfrak{H C} \log \frac{\mu+C_{1}+C_{2}}{\mu+C_{1}-C_{2}}\right) \mathfrak{H C} \frac{C_{2}}{\mu+C_{1}}\left(\alpha_{22} \alpha_{12} \theta_{2} \theta_{3}+\mathfrak{C C} \alpha_{12} \theta_{2} \theta_{3}\right) \\
& \left.\left.\cdot\left[1-C_{2}^{2}\left(\mu+C_{1}\right)^{-2}\right]^{-1 / 2} \exp \left(\frac{1}{2} \nVdash \log \frac{\mu+C_{1}+C_{2}}{\mu+C_{1}-C_{2}}\right)\right]\right\} \text {, }
\end{aligned}
$$

and

$$
\begin{gathered}
S=\frac{1}{2}\left(1-C_{0}\right)^{-1}\left\{\frac{\mu}{\mu-C_{1}}\left[1-C_{2}^{2}\left(\mu-C_{1}\right)^{-2}\right]^{-1 / 2} \exp \left(-\frac{1}{2} \mathcal{H C} \log \frac{\mu-C_{1}+C_{2}}{\mu-C_{1}-C_{2}}\right)\right. \\
\left.+\frac{\mu}{\mu+C_{1}}\left[1-C_{2}^{2}\left(\mu+C_{1}\right)^{-2}\right]^{-1 / 2} \exp \left(\frac{1}{2} \mathcal{H C} \log \frac{\mu+C_{1}+C_{2}}{\mu+C_{1}-C_{2}}\right)\right\} .
\end{gathered}
$$


In (18), the following notations have been used:

$$
\begin{gathered}
C_{0}=\alpha_{21} \theta_{1} \theta_{4}\left\{\alpha _ { 1 2 } \theta _ { 2 } \theta _ { 3 } \left[\alpha_{11} \alpha_{22}-\alpha_{12} \theta_{2} \theta_{3}\left(\alpha_{11}+\alpha_{22}\right)^{2}\left(4 \alpha_{12} \theta_{2} \theta_{3}\right.\right.\right. \\
\left.\left.\left.-2 \mathcal{C}\left[\alpha_{11}-\alpha_{22}\right] \alpha_{12} \theta_{2} \theta_{3}\right)^{-1}\right]+\frac{1}{2} \mathfrak{F}\left[\alpha_{11}-\alpha_{22}\right] \alpha_{12} \theta_{2} \theta_{3}\right\}, \\
C_{1}=-\frac{1}{2}\left(\alpha_{11}+\alpha_{22}\right) \alpha_{12} \theta_{2} \theta_{3}, \\
C_{2}=\alpha_{12} \theta_{2} \theta_{3}-\frac{1}{2} \mathfrak{C}\left[\alpha_{11}-\alpha_{22}\right] \alpha_{12} \theta_{2} \theta_{3} .
\end{gathered}
$$

If, instead of (16), the following alternative assumption is made:

$$
\begin{aligned}
\alpha_{12}= & \theta_{2}^{-1} \theta_{3}^{-1}\left(\alpha_{21} \theta_{1} \theta_{4}-\frac{1}{2} \mathcal{H C}\left[\alpha_{22}-\alpha_{11}\right] \alpha_{21} \theta_{1} \theta_{4}\right)\left\{\mu^{2}-\frac{1}{4} \alpha_{21}^{2} \theta_{1}^{2} \theta_{4}^{2}\left(\alpha_{22}-\alpha_{11}\right)^{2}\right. \\
& \left.\quad+\frac{1}{2} \alpha_{21} \theta_{1} \theta_{4}\left(1-\alpha_{11} \alpha_{22}\right) \mathcal{H C}\left[\alpha_{22}-\alpha_{11}\right] \alpha_{21} \theta_{1} \theta_{4}-\frac{1}{4}\left(\mathcal{F C}\left[\alpha_{22}-\alpha_{11}\right] \alpha_{21} \theta_{1} \theta_{4}\right)^{2}\right\}^{-1},
\end{aligned}
$$

(which is obtained from (16) by interchanging $\alpha_{11}$ and $\alpha_{22}, \alpha_{12} \theta_{2} \theta_{3}$ and $\alpha_{21} \theta_{1} \theta_{4}$ ), then

$$
\begin{gathered}
A=\left[\begin{array}{cc}
\theta_{1}-\alpha_{11} \alpha_{12}\left(1-\alpha_{11}^{2}\right)^{-1} \theta_{2} Q^{\prime}+\theta_{1} \mathcal{F} \alpha_{12} \theta_{2} \theta_{3} Q^{\prime} & \alpha_{11} \alpha_{12}\left(1-\alpha_{11}^{2}\right)^{-1} \theta_{2} P^{\prime}-\theta_{1} \mathcal{F} \alpha_{12} \theta_{2} \theta_{3} P^{\prime} \\
-\theta_{2} Q^{\prime} & \theta_{2} P^{\prime}
\end{array}\right], \\
B=\left[\begin{array}{cc}
\theta_{3} S^{\prime} & \alpha_{22} \alpha_{12}\left(1-\alpha_{22}^{2}\right)^{-1} \theta_{3} S^{\prime}+\theta_{4} \mathcal{F} \alpha_{12} \theta_{2} \theta_{3} S^{\prime} \\
\theta_{3} R^{\prime} & \theta_{4}+\alpha_{22} \alpha_{12}\left(1-\alpha_{22}^{2}\right)^{-1} \theta_{3} R^{\prime}+\theta_{4} \mathcal{H} \alpha_{12} \theta_{2} \theta_{3} R^{\prime}
\end{array}\right],
\end{gathered}
$$

where the $\mathrm{P}^{\prime}, Q^{\prime}, R^{\prime}$ and $S^{\prime}$ are obtained from $P, Q, R$ and $S$ with the same interchanges. Note that for $\alpha_{11}=\alpha_{22}$, both (16) and (20) are reduced to $\alpha_{12}=$ const. $\alpha_{21}$. This particular case for coupled Wiener-Hopf equations is well known. A detailed account of the derivations of (17) and (21) is given in Appendix B.

So far a procedure has been found to give the required solutions in closed forms for two special cases. A similar procedure can be used to give information of a somewhat different kind for

$$
\alpha_{11}=\alpha_{22}=0 .
$$

When (22a) holds, it is shown in Appendix $\mathrm{C}$ that

$$
\left[\begin{array}{ll}
A_{11} & A_{12} \\
A_{21} & A_{22}
\end{array}\right]=\left[\begin{array}{rr}
B_{22} & -B_{12} \\
-B_{21} & B_{11}
\end{array}\right]=\left[\begin{array}{cc}
B_{22} & -B_{12} \\
-\Im C \alpha_{21} B_{22} & 1+\Im C \alpha_{21} B_{12}
\end{array}\right],
$$

Hence both $A$ and $B$ are completely determined by $B_{22}$ and $B_{12}$. The result is of the following nature. Consider two systems of coupled Wiener-Hopf integral equations with $\alpha_{11}=\alpha_{22}=\alpha_{11}^{\prime}=\alpha_{22}^{\prime}=0$ :

$$
\phi+\alpha \mathfrak{H} \phi=h, \quad \phi^{\prime}+\alpha^{\prime} \mathfrak{F} \phi^{\prime}=h^{\prime} .
$$

If $A$ and hence $B$ is known for the first system of equations, then the solution for the second system can be written in closed form provided that $\alpha^{\prime}$ is a certain specific function of $\alpha$. More precisely, if $\alpha_{12}^{\prime}$ and $\alpha_{21}^{\prime}$ are given by

$$
\begin{gathered}
\alpha_{12}^{\prime}=\mathfrak{F C} \tanh \left(\frac{1}{2} \mathfrak{H C} \log \frac{1+\alpha_{12}}{1-\alpha_{12}}\right) \\
\alpha_{21}^{\prime}=\left(\alpha_{21}-\alpha_{12}\right)\left(1-\alpha_{12}^{2}\right)^{-1} \cosh ^{2}\left(\frac{1}{2} \mathfrak{H C} \log \frac{1+\alpha_{12}}{1-\alpha_{12}}\right)\left\{1-\alpha_{12}\left(\alpha_{21}-\alpha_{12}\right)\left(1-\alpha_{12}^{2}\right)^{-1}\right. \\
\left.+\left(\alpha_{21}-\alpha_{12}\right)\left(1-\alpha_{12}^{2}\right)^{-1 / 2} \cosh \left(\frac{1}{2} \mathfrak{H C} \log \frac{1+\alpha_{12}}{1-\alpha_{12}}\right) \operatorname{HC} \tanh \left(\frac{1}{2} \mathfrak{H C} \log \frac{1+\alpha_{12}}{1-\alpha_{12}}\right)\right\}^{-1},
\end{gathered}
$$


then the $B_{22}^{\prime}$ and $B_{12}^{\prime}$ that correspond to $\alpha_{12}^{\prime}$ and $\alpha_{21}^{\prime}$ are found in Appendix $\mathrm{C}$ to be

$$
\begin{aligned}
& B_{22}^{\prime}=D B_{22} \\
& B_{12}^{\prime}=D B_{12},
\end{aligned}
$$

where

$$
\begin{aligned}
& D=\left\{\cosh \left(\frac{1}{2} \mathfrak{F C} \log \frac{1+\alpha_{12}}{1-\alpha_{12}}\right)\right\}^{-1}\left(1-\alpha_{12}^{2}\right)^{-1 / 2}\left\{1-\alpha_{12}\left(\alpha_{21}-\alpha_{12}\right)\left(1-\alpha_{12}^{2}\right)^{-1}\right. \\
& \left.+\left(\alpha_{21}-\alpha_{12}\right)\left(1-\alpha_{12}^{2}\right)^{-1 / 2} \cosh \left(\frac{1}{2} \mathfrak{H C} \log \frac{1+\alpha_{12}}{1-\alpha_{12}}\right) \mathcal{H C t a n h}\left(\frac{1}{2} \mathcal{H C} \log \frac{1+\alpha_{12}}{1-\alpha_{12}}\right)\right\}^{-1} .
\end{aligned}
$$

A similar result can be obtained by interchanging $\alpha_{12}$ and $\alpha_{21}$ in (23) and (25) for the calculation of $B_{11}$ and $B_{21}$ instead of $B_{22}$ and $B_{12}$. This method of generating new equations can be applied repeatedly.

4. Relation to conventional procedure. In coupled equations, the Wiener-Hopf method becomes difficult since the kernel to be split is now a matrix. However, for the classes of equations considered in the present paper, the splitting can be performed with the help of the $B$ matrix. If $\phi(z)$ is defined by [5]

$$
\phi(z)=\frac{1}{2 \pi i} \int_{-\infty}^{\infty} \frac{\phi(t)}{t-z} d t
$$

for complex $z$, and if $\phi^{+}(x)$ be the limit of $\phi(z)$ as $z$ approaches the real axis from the upper half-plane and if $\phi^{-}(x)$ is the limit of $\phi(z)$ as $z$ approaches the real axis from the lower half-plane, (13) becomes

$$
G(x) \phi^{+}(x)-\phi^{-}(x)=[1-\alpha(x)]^{-1} h(x),
$$

where $G(x)=[1-\alpha(x)]^{-1}[1+\alpha(x)]$. The usual Wiener-Hopf procedure involves a definition of $G^{+}(x)$ and $G^{-}(x)$ such that $G^{+}(x)$ can be analytically continued into the entire upper half-plane, $G^{-}(x)$ can be analytically continued into the entire lower halfplane, and $G(x)=\left[G^{-}(x)\right]^{-1} G^{+}(x)$. On the other hand, it follows from (14) that $B=1+\Im B C$, or $B(1+\alpha)=1+B \alpha+\Im B \alpha$. Hence, $B(1+\alpha)$ can be analytically continued into the entire upper half-plane. Indeed it is permissible to make the following identification

$$
G^{+}=B(1+\alpha), \quad G^{-}=B(1-\alpha) .
$$

Once $G^{+}$and $G^{-}$are known, the rest of the Wiener-Hopf procedure involves only additive decompositions, which are no more complicated in general than in the case of a single integral equation.

5. Conclusions. A method of iteration has been used to study the Wiener-Hopf integral equations. In the case of a single equation, the usual solution is obtained. In the case of two coupled equations, a certain class, which appears to be difficult to construct by the usual method of Wiener and Hopf without knowing the answer in advance, can be solved in closed form. Even though it is presumably possible to obtain the results given here without recourse to the method of iteration, this method seems very powerful for obtaining new results and is in this sense a useful technique. The two special cases considered in detail here by no means exhaust all the possibilities, although it does not 
seem likely that the present procedure is capable of producing a solution for the general case in a closed form.

Acknowledgments. For helpful discussions we are greatly indebted to Professor Bernard Budiansky, Professor George F. Carrier, and Professor Ronold W. P. King. The work of the first-named author was supported by the Advanced Research Project Agency (Project SD-88) and that of the second named author by the Alfred P. Sloan Foundation.

\section{APPENDIX A}

The direct summation of (11) is possible if in $\mathfrak{F C}^{\mathfrak{C}} \alpha \mathfrak{H} \alpha \cdots \mathfrak{F} \alpha$ with $n \alpha^{\prime}$ 's, the coefficient $K\left(a, b_{i}\right)$ of the general term $\alpha^{a} \Pi_{i} \mathfrak{H C} \alpha^{b_{i}}$ is known, where $a$ is even, $b_{i}$ 's are odd, and $a+\sum_{i} b_{i}=n$. By repeated application of (7), the following relation can be established,

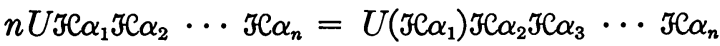

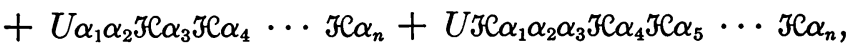

where $U$ is an operator which permutes all the $\alpha$ 's and in the last term also symmetrizes with respect to the position of $\alpha_{1} \alpha_{2} \alpha_{3} \cdots$. For example

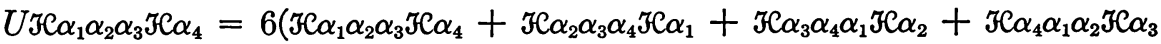

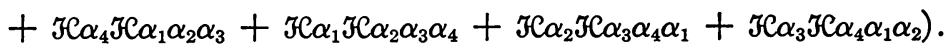

In the following calculation, the $\alpha_{i}$ 's in (A1) are equal. Define

$$
F\left(a, b_{i}\right)=K\left(a, b_{i}\right) V a ! \Pi_{i} b_{i} !,
$$

where $V$ is the order of the symmetry group of the $b_{i}$ 's; and the convention is used that $F\left(a, b_{i}\right)=0$ if $a<0$, and that $b_{j}$ is omitted from the arguments of $F\left(a, b_{i}\right)$ if $b_{i}<0$. For the general term under consideration, (A1) gives

$$
\begin{aligned}
& n F\left(a, b_{i}\right)=\sum_{b_{i}=1} F\left(a, b_{i}-2 \delta_{i j}\right)+a(a-1) F\left(a-2, b_{i}\right) \\
& \quad+\left\{a(a-1)(a-2) F\left(a-2, b_{i}\right)+\sum_{i} b_{i}\left(b_{i}-1\right)\left(b_{i}-2\right) F\left(a, b_{i}-2 \delta_{i j}\right)\right\} \\
& =\sum_{b_{j}=1} F\left(a, b_{i}-2 \delta_{i j}\right)+a(a-1)^{2} F\left(a-2, b_{i}\right) \\
& \quad+\sum_{i} b_{i}\left(b_{i}-1\right)\left(b_{i}-2\right) F\left(a, b_{i}-2 \delta_{i j}\right) .
\end{aligned}
$$

Let $F\left(a, b_{i}\right)=G\left(a, b_{i}\right) \Pi_{b_{i}>0}\left(b_{i}-1\right)$ !, then (A4) gives

$$
\left(a+\sum_{i} b_{j}\right) G\left(a, b_{i}\right)=a(a-1)^{2} G\left(a-2, b_{i}\right)+\sum_{i} b_{j} G\left(a, b_{i}-2 \delta_{i j}\right) .
$$

Next, let $G\left(0, b_{i}\right)=H\left(0, b_{i}\right)$ and $G\left(a, b_{i}\right)=[(a-1) ! !]^{2} H\left(a, b_{i}\right)$ for $a \geq 2$, where $(a-1) ! !=(a-1)(a-3)(a-5) \cdots 3 \cdot 1$. Then (A5) gives, for $a \geq 0$ and all $b_{i}>0$,

$$
\left(a+\sum_{i} b_{i}\right) H\left(a, b_{i}\right)=a H\left(a-2, b_{i}\right)+\sum_{i} b_{i} H\left(a, b_{i}-2 \delta_{i i}\right),
$$


which is satisfied if, and only if, $H\left(a, b_{i}\right)=$ const. From the condition $F(0)=1$, it follows that $H\left(a, b_{i}\right)=1$. Hence, for $a \geq 0$ and all $b_{i}>0$,

$$
K\left(a, b_{i}\right)= \begin{cases}\left(\prod_{i} b_{i}\right)^{-1} V^{-1}, & \text { for } a=0, \\ {[(a-1) ! !]^{2}(a !)^{-1}\left(\prod_{i} b_{i}\right)^{-1} V^{-1},} & \text { for } a \geq 2 .\end{cases}
$$

Equation (12) follows directly from (A7).

\section{APPENDIX B}

The matrix equations (11a) and (14) are the same as the following eight equations for the elements of the matrices $A$ and $B$ :

$$
\begin{aligned}
& A_{11}=1-\operatorname{HC} \alpha_{11}+\operatorname{HC} \alpha_{1 i} \operatorname{HC} \alpha_{i 1}-\operatorname{FC} \alpha_{1 i} \operatorname{HC} \alpha_{i j} \mathfrak{H} \alpha_{i 1}+\cdots,
\end{aligned}
$$

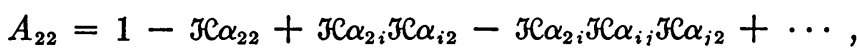

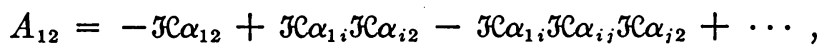

$$
\begin{aligned}
& A_{21}=-\mathfrak{H} \alpha_{21}+\mathcal{H} \mathcal{C} \alpha_{2 i} \mathfrak{H} \alpha_{i 1}-\mathcal{H} \mathcal{C} \alpha_{2 i} \mathcal{H} \mathcal{C} \alpha_{i j} \mathcal{H} \alpha_{j 1}+\cdots \text {, }
\end{aligned}
$$

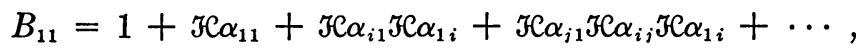

$$
\begin{aligned}
& B_{22}=1+\mathfrak{H} \alpha_{22}+\mathfrak{H} \alpha_{i 2} \mathfrak{H} \alpha_{2 i}+\mathfrak{H} \alpha_{j 2} \mathfrak{H} \alpha_{i j} \mathfrak{H} \alpha_{2 i}+\cdots,
\end{aligned}
$$

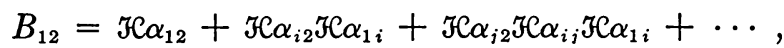

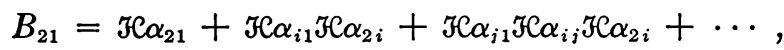

where the summation convention for repeated subscripts has been used. In (7), ( ) has been used to denote separate factors; and it is convenient to define the [ ] notation:

$$
\left[\mathcal{H C} \alpha_{1}\right] \mathcal{H C} \alpha_{2} \equiv \mathcal{H C} \alpha_{1} \mathcal{H C} \alpha_{2}
$$

With the notation (B3), (B1) and (B2) are expanded as

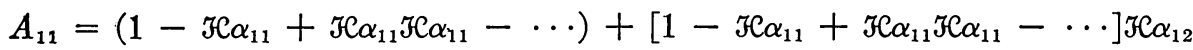

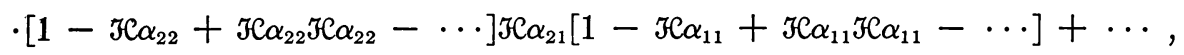

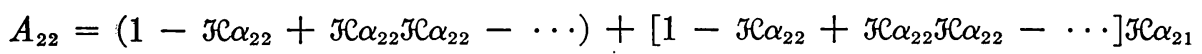

$$
\begin{aligned}
& \cdot\left[1-\mathcal{H} \alpha_{11}+\mathcal{H} \alpha_{11} \mathfrak{H C} \alpha_{11}-\cdots\right] \mathcal{H C} \alpha_{12}\left[1-\mathcal{H} \alpha_{22}+\mathcal{H} \alpha_{22} \mathcal{H} \alpha_{22}-\cdots\right]+\cdots, \\
& A_{12}=-\left[1-\mathcal{H} C \alpha_{11}+\mathcal{H} \alpha_{11} \mathfrak{H C} \alpha_{11}-\cdots\right] \mathcal{H C} \alpha_{12}\left[1-\mathcal{H} C \alpha_{22}+\mathcal{H} C \alpha_{22} \mathfrak{H C} \alpha_{22}-\cdots\right]
\end{aligned}
$$

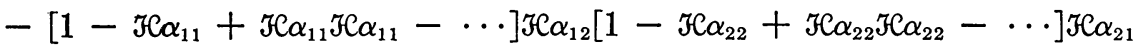

$$
\begin{aligned}
& \cdot\left[1-\mathcal{H} \alpha_{11}+\mathcal{H} \alpha_{11} \mathfrak{H C} \alpha_{11}-\cdots\right] \mathcal{H C} \alpha_{12}\left[1-\mathcal{H} \alpha_{22}+\mathcal{H} \alpha_{22} \mathcal{H} \alpha_{22}-\cdots\right]-\cdots, \\
& A_{21}=-\left[1-\mathcal{H} \alpha_{22}+\mathcal{H} \alpha_{22} \mathcal{H} \alpha_{22}-\cdots\right] \mathcal{H C} \alpha_{21}\left[1-\mathcal{H} \alpha_{11}+\mathcal{H} \alpha_{11} \mathfrak{H C} \alpha_{11}-\cdots\right]
\end{aligned}
$$

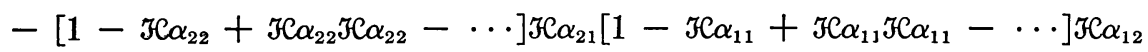

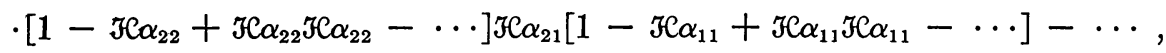

$$
\begin{aligned}
& B_{11}=\left(1+\mathcal{H} \alpha_{11}+\mathcal{H} \alpha_{11} \mathcal{H C} \alpha_{11}+\cdots\right)+\left[1+\mathcal{H} \mathcal{C} \alpha_{11}+\mathcal{H} \mathcal{C} \alpha_{11} \mathcal{H} \alpha_{11}+\cdots\right] \mathcal{H} \alpha_{21}
\end{aligned}
$$

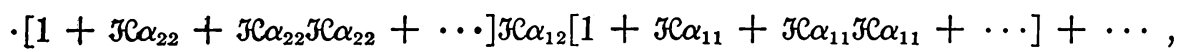




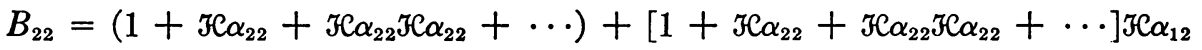

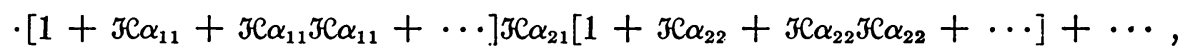

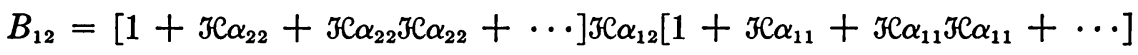

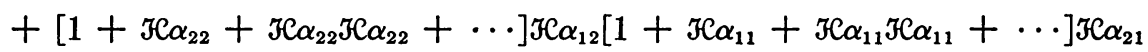

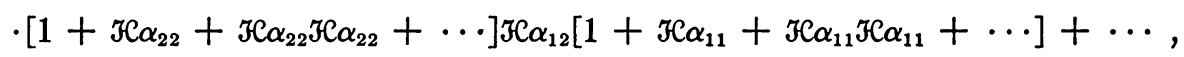

$$
\begin{aligned}
& B_{21}=\left[1+\mathcal{F} \alpha_{11}+\mathcal{F} \alpha_{11} \mathcal{F} \alpha_{11}+\cdots\right] \mathcal{F} \alpha_{21}\left[1+\mathcal{F} \alpha_{22}+\mathcal{F} \alpha_{22} \mathcal{T} \alpha_{22}+\cdots\right]
\end{aligned}
$$

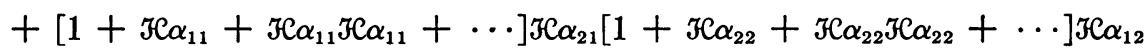

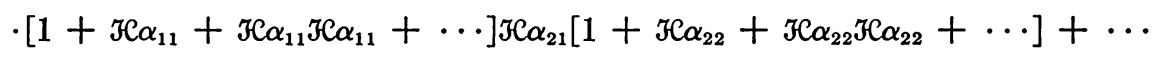

With the use of the system of equalities indicated by (7), (8) and (9), the operators

$$
\begin{aligned}
& {\left[1-\mathcal{F} \alpha_{11}+\mathcal{F} \alpha_{11} \mathcal{F} \alpha_{11}-\cdots\right] \mathcal{F}=-\alpha_{11} \theta_{1} \theta_{3}+\theta_{1} \mathcal{F} \theta_{3},} \\
& {\left[1-\mathfrak{H C} \alpha_{22}+\mathfrak{H C} \alpha_{22} \mathfrak{H C} \alpha_{22}-\cdots\right] \mathfrak{H C}=-\alpha_{22} \theta_{2} \theta_{4}+\theta_{2} \mathfrak{H} \theta_{4},} \\
& {\left[1+\mathfrak{H C} \alpha_{11}+\mathfrak{H C} \alpha_{11} \mathfrak{H C} \alpha_{11}+\cdots\right] \mathcal{H}=\alpha_{11} \theta_{1} \theta_{3}+\theta_{3} \mathfrak{H} \theta_{1},}
\end{aligned}
$$

and

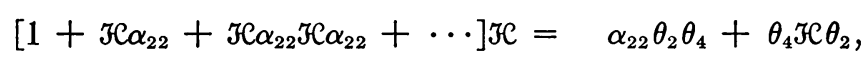

where the $\theta_{i}$ 's are defined in (15). The substitution of (B6) into (B4) and (B5) gives

$$
\begin{aligned}
& A_{11}=\theta_{1}\left(1+\left[-\alpha_{11} \alpha_{12} \theta_{2} \theta_{3}+\mathcal{F} \alpha_{12} \theta_{2} \theta_{3}\right]\left[-\alpha_{22} \alpha_{21} \theta_{1} \theta_{4}+\mathcal{F} \alpha_{21} \theta_{1} \theta_{4}\right]+\cdots\right) \text {, } \\
& A_{22}=\theta_{2}\left(1+\left[-\alpha_{22} \alpha_{21} \theta_{1} \theta_{4}+\mathcal{F} \alpha_{21} \theta_{1} \theta_{4}\right]\left[-\alpha_{11} \alpha_{12} \theta_{2} \theta_{3}+\mathcal{H} \alpha_{12} \theta_{2} \theta_{3}\right]+\cdots\right) \text {, } \\
& A_{12}=-\theta_{1}\left(-\alpha_{11} \alpha_{12} \theta_{2} \theta_{3}+\mathfrak{H} \alpha_{12} \theta_{2} \theta_{3}+\left[-\alpha_{11} \alpha_{12} \theta_{2} \theta_{3}+\mathfrak{T C} \alpha_{12} \theta_{2} \theta_{3}\right]\right. \\
& \left.\cdot\left[-\alpha_{22} \alpha_{21} \theta_{1} \theta_{4}+\mathcal{F} \alpha_{21} \theta_{1} \theta_{4}\right]\left[-\alpha_{11} \alpha_{12} \theta_{2} \theta_{3}+\mathcal{F} \alpha_{12} \theta_{2} \theta_{3}\right]+\cdots\right), \\
& A_{21}=-\theta_{2}\left(-\alpha_{22} \alpha_{21} \theta_{1} \theta_{4}+\mathcal{H} \alpha_{21} \theta_{1} \theta_{4}+\left[-\alpha_{22} \alpha_{21} \theta_{1} \theta_{4}+\mathcal{H} \alpha_{21} \theta_{1} \theta_{4}\right]\right. \\
& \left.\cdot\left[-\alpha_{11} \alpha_{12} \theta_{2} \theta_{3}+\mathcal{F} \alpha_{12} \theta_{2} \theta_{3}\right]\left[-\alpha_{22} \alpha_{21} \theta_{1} \theta_{4}+\mathcal{F} \alpha_{21} \theta_{1} \theta_{4}\right]+\cdots\right), \\
& B_{11}=\theta_{3}\left(1+\left[\alpha_{11} \alpha_{21} \theta_{1} \theta_{4}+\mathcal{F} \alpha_{21} \theta_{1} \theta_{4}\right]\left[\alpha_{22} \alpha_{12} \theta_{2} \theta_{3}+\mathcal{H} \alpha_{12} \theta_{2} \theta_{3}\right]+\cdots\right) \text {, } \\
& B_{22}=\theta_{4}\left(1+\left[\alpha_{22} \alpha_{12} \theta_{2} \theta_{3}+\mathcal{F} \alpha_{12} \theta_{2} \theta_{3}\right]\left[\alpha_{11} \alpha_{21} \theta_{1} \theta_{4}+\mathcal{F} \alpha_{21} \theta_{1} \theta_{4}\right]+\cdots\right) \text {, } \\
& B_{12}=\theta_{4}\left(\alpha_{22} \alpha_{12} \theta_{2} \theta_{3}+\mathcal{H} \alpha_{12} \theta_{2} \theta_{3}+\left[\alpha_{22} \alpha_{12} \theta_{2} \theta_{3}+\mathcal{H} \alpha_{12} \theta_{2} \theta_{3}\right]\right. \\
& \left.\cdot\left[\alpha_{11} \alpha_{21} \theta_{1} \theta_{4}+\Im \mathcal{C} \alpha_{21} \theta_{1} \theta_{4}\right]\left[\alpha_{22} \alpha_{12} \theta_{2} \theta_{3}+\Im \mathcal{C} \alpha_{12} \theta_{2} \theta_{3}\right]+\cdots\right), \\
& B_{21}=\theta_{3}\left(\alpha_{11} \alpha_{21} \theta_{1} \theta_{4}+\mathcal{F} \alpha_{21} \theta_{1} \theta_{4}+\left[\alpha_{11} \alpha_{21} \theta_{1} \theta_{4}+\mathcal{F} \alpha_{21} \theta_{1} \theta_{4}\right]\right. \\
& \left.\cdot\left[\alpha_{22} \alpha_{12} \theta_{2} \theta_{3}+\mathcal{F} \mathcal{C} \alpha_{12} \theta_{2} \theta_{3}\right]\left[\alpha_{11} \alpha_{21} \theta_{1} \theta_{4}+\mathcal{F} \mathcal{C} \alpha_{21} \theta_{1} \theta_{4}\right]+\cdots\right) \text {. }
\end{aligned}
$$

Note that $A_{22}, A_{21}, B_{11}$ and $B_{21}$ can be expressed in terms of $A_{11}, A_{12}, B_{22}$ and $B_{12}$; and vice versa. Therefore, it is sufficient to consider one of the two sets, and the latter set is studied in detail here. Let $A_{11}=\theta_{1} P, A_{12}=-\theta_{1} Q, B_{22}=\theta_{4} S$, and $B_{12}=\theta_{4} R$ so that $P, Q, R$ and $S$ satisfy the following four integral equations respectively:

$P=1+\left[-\alpha_{11} \alpha_{12} \theta_{2} \theta_{3}+\mathcal{F} \alpha_{12} \theta_{2} \theta_{3}\right]\left[-\alpha_{22} \alpha_{21} \theta_{1} \theta_{4}+\mathcal{F} \alpha_{21} \theta_{1} \theta_{4}\right] P$, 
$Q=-\alpha_{11} \alpha_{12} \theta_{2} \theta_{3}+\Im{ }^{C} \alpha_{12} \theta_{2} \theta_{3}+\left[-\alpha_{11} \alpha_{12} \theta_{2} \theta_{3}+{ } C \alpha_{12} \theta_{2} \theta_{3}\right]$

$$
\cdot\left[-\alpha_{22} \alpha_{21} \theta_{1} \theta_{4}+\mathcal{F C}_{21} \theta_{1} \theta_{4}\right] Q,
$$

$R=\alpha_{22} \alpha_{12} \theta_{2} \theta_{3}+\Im \mathcal{C} \alpha_{12} \theta_{2} \theta_{3}+\left[\alpha_{22} \alpha_{12} \theta_{2} \theta_{3}+\Im \mathcal{C} \alpha_{12} \theta_{2} \theta_{3}\right]\left[\alpha_{11} \alpha_{21} \theta_{1} \theta_{4}+\Im C \alpha_{21} \theta_{1} \theta_{4}\right] R$,

$S=1+\left[\alpha_{22} \alpha_{12} \theta_{2} \theta_{3}+\operatorname{TC}_{12} \theta_{2} \theta_{3}\right]\left[\alpha_{11} \alpha_{21} \theta_{1} \theta_{4}+{ }^{C} \alpha_{21} \theta_{1} \theta_{4}\right] S$.

It is now clear that the separation of the elements of $A$ and $B$ into two sets has been based on the similarity of the integral equations (B9). In (B9a), by (7),

$$
\begin{gathered}
{\left[-\alpha_{11} \alpha_{12} \theta_{2} \theta_{3}+\mathcal{H} \alpha_{12} \theta_{2} \theta_{3}\right]\left[-\alpha_{22} \alpha_{21} \theta_{1} \theta_{4}+\mathcal{F} \alpha_{21} \theta_{1} \theta_{4}\right] P=\left(1+\alpha_{11} \alpha_{22}\right) \alpha_{12} \alpha_{21} \theta_{1} \theta_{2} \theta_{3} \theta_{4} P} \\
+\left(-\alpha_{11} \alpha_{12} \theta_{2} \theta_{3}+\Im \mathcal{C} \alpha_{12} \theta_{2} \theta_{3}\right)\left(\mathcal{F C} \alpha_{21} \theta_{1} \theta_{4} P\right)+\mathcal{F} \alpha_{21} \theta_{1} \theta_{4} P\left[-\alpha_{22} \alpha_{12} \theta_{2} \theta_{3}-\Im \mathcal{C} \alpha_{12} \theta_{2} \theta_{3}\right] .
\end{gathered}
$$

It is desired to write this expression in the form $C_{0} P+\left[C_{1}+\Im_{C C} C_{2}\right]\left[C_{3}+\Im_{C} C_{4}\right] P$ such that $C_{1} / C_{3}=C_{2} / C_{4}$, where the $C_{i}$ 's are to be determined. Again, by (7),

$$
\begin{aligned}
& \mathrm{C}_{0} P+\left[\mathrm{C}_{1}+\mathrm{FCC}_{2}\right]\left[\mathrm{C}_{3}+\mathfrak{F C C}_{4}\right] P \\
& =\left(C_{0}+C_{1} C_{3}+C_{2} C_{4}\right) P+\left(C_{1}+F_{C} C_{2}\right)\left(F_{C} C_{4} P\right)+\mathfrak{F C P}\left[C_{2} C_{3}-C_{4} \mathcal{F C}_{2}\right] .
\end{aligned}
$$

A comparison of (B10) and (B11) gives that

$$
\begin{aligned}
& C_{4}=\alpha_{21} \theta_{1} \theta_{4}, \\
& C_{1}+\mathcal{H C} C_{2}=-\alpha_{11} \alpha_{12} \theta_{2} \theta_{3}+\mathcal{F} \alpha_{12} \theta_{2} \theta_{3}, \\
& C_{2} C_{3}-C_{4} \mathcal{H C} C_{2}=\alpha_{21} \theta_{1} \theta_{4}\left(-\alpha_{22} \alpha_{12} \theta_{2} \theta_{3}-\mathcal{F C} \alpha_{12} \theta_{2} \theta_{3}\right), \\
& C_{0}+C_{1} C_{3}+C_{2} C_{4}=\left(1+\alpha_{11} \alpha_{22}\right) \alpha_{12} \alpha_{21} \theta_{1} \theta_{2} \theta_{3} \theta_{4} .
\end{aligned}
$$

Together with $C_{1} / C_{3}=C_{2} / C_{4}$ and $\phi=\mathcal{F C}^{2} \phi$, (B12) can be solved for the $C_{i}$ 's as given in (19), (B12a) and

$$
C_{3}=-\frac{1}{2}\left(\alpha_{11}+\alpha_{22}\right) \alpha_{12} \alpha_{21} \theta_{1} \theta_{2} \theta_{3} \theta_{4}\left(\alpha_{12} \theta_{2} \theta_{3}-\frac{1}{2} \mathcal{C}\left[\alpha_{11}-\alpha_{22}\right] \alpha_{12} \theta_{2} \theta_{3}\right)^{-1} \text {. }
$$

Of course, a similar substitution is possible for (B9b); furthermore, it happens that both (B9c) and (B9d) can be likewise substituted. After some rearrangement, (B9) may be expressed as

$$
\begin{aligned}
& \left(1-C_{0}\right) P=1+\left[C_{1}+\mathcal{F C}_{2}\right]\left[C_{3}+\mathcal{F C C}_{4}\right] P, \\
& \left(1-C_{0}\right) Q=-\alpha_{11} \alpha_{12} \theta_{2} \theta_{3}+\mathfrak{H C}_{12} \theta_{2} \theta_{3}+\left[C_{1}+\mathfrak{F C}_{2}\right]\left[C_{3}+\mathfrak{H C C}_{4}\right] Q \text {, } \\
& \left(1-C_{0}\right) R=\alpha_{22} \alpha_{12} \theta_{2} \theta_{3}+\mathfrak{F C}_{12} \theta_{2} \theta_{3}+\left[C_{1}-\mathfrak{F C C}_{2}\right]\left[C_{3}-\mathfrak{F C C}_{4}\right] R,
\end{aligned}
$$

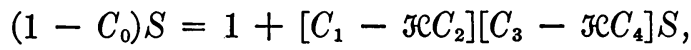

which can all be solved in closed form under one restrictive condition, i.e.

$$
\frac{C_{1}\left(1-C_{0}\right)}{C_{3}}=\mu^{2},
$$

where $\mu$ is any constant. This is used to solve for $\alpha_{21}$ in terms of $\alpha_{11}, \alpha_{22}$, and $\alpha_{12}$, as shown in (16). Note that this simple result follows from the fact that the operator $\left[\alpha_{22} \alpha_{12} \theta_{2} \theta_{3}+{ }^{3} \alpha_{12} \theta_{2} \theta_{3}\right]\left[\alpha_{11} \alpha_{21} \theta_{1} \theta_{4}+{ }^{C} \alpha_{21} \theta_{1} \theta_{4}\right]$ can be obtained from [- $\alpha_{11} \alpha_{12} \theta_{2} \theta_{3}+$ $\left.{ }^{3}{ }^{3} \alpha_{12} \theta_{2} \theta_{3}\right]\left[-\alpha_{22} \alpha_{21} \theta_{1} \theta_{4}+{ }^{3 C} \alpha_{21} \theta_{1} \theta_{4}\right]$ by the substitutions of $\alpha_{11}$ by $-\alpha_{22}$, and $\alpha_{22}$ by 
$-\alpha_{11}$, which also lead to the replacements of $C_{0}$ by $C_{0}, C_{1}$ by $-C_{1}, C_{2}$ by $C_{2}, C_{3}$ by $-C_{3}$, and $C_{4}$ by $C_{4}$.

When (B15) holds, the solution of (B14) is given by (18).

By consideration of $A_{22}, A_{21}, B_{11}$ and $B_{21}$, the restrictive condition (16) changes to (20), and the matrices $A$ and $B$ are expressed by (21) instead of (17).

\section{APPENDIX C}

When the system of two coupled Wiener-Hopf equations contains only cross terms, i.e. $\alpha_{11}=\alpha_{22}=0,(\mathrm{~B} 1)$ and (B2) are reduced to

$$
\begin{aligned}
& A_{11}=B_{22}=1+\Im \mathcal{C} \alpha_{12} \Im C \alpha_{21}+\Im \mathcal{C} \alpha_{12} \Im C \alpha_{21} \Im C \alpha_{12} \Im C \alpha_{21}+\cdots, \\
& A_{22}=B_{11}=1+\Im \mathcal{C} \alpha_{21} \Im C \alpha_{12}+\Im \mathcal{C} \alpha_{21} \Im C \alpha_{12} \Im C \alpha_{21} \Im C \alpha_{12}+\cdots,
\end{aligned}
$$

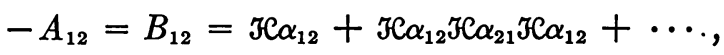

$$
\begin{aligned}
& -A_{21}=B_{21}=\mathfrak{H C} \alpha_{21}+\mathcal{H C} \alpha_{21} \mathfrak{H C} \alpha_{12} \mathfrak{H} \alpha_{21}+\cdots \text {. }
\end{aligned}
$$

In this case, $B_{22}$ and $B_{12}$ form one set while $B_{11}$ and $B_{21}$ form another. Here, the former set will be considered in detail. Let $\alpha_{21}=\alpha_{12}+\varepsilon$; then

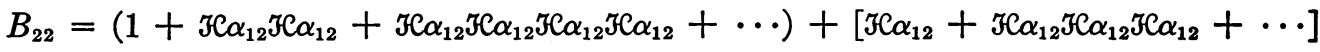

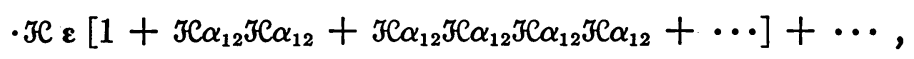

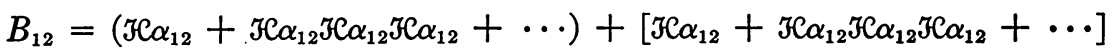

$$
\begin{aligned}
& \text {. } \mathcal{H C} \varepsilon\left[\mathcal{H C} \alpha_{12}+\mathcal{H} \alpha_{12} \mathcal{H} \alpha_{12} \mathcal{H} \alpha_{12}+\cdots\right]+\cdots,
\end{aligned}
$$

which can be alternatively written as

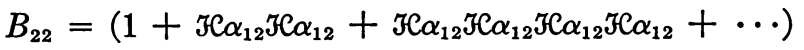

$$
\begin{aligned}
& +\left[\mathfrak{H C} \alpha_{12}+\mathcal{H} \alpha_{12} \mathfrak{H} \alpha_{12} \mathfrak{H} \alpha_{12}+\cdots\right] \mathcal{H C} \varepsilon B_{22}
\end{aligned}
$$

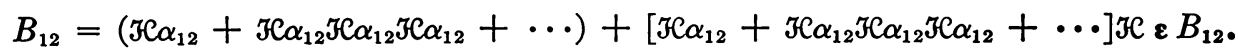

Similar to (B6), with

$$
\begin{aligned}
& N_{1}=\mathcal{F} \alpha_{12}+\mathcal{F} \alpha_{12} \mathcal{F} \alpha_{12} \mathcal{F} \alpha_{12}+\cdots=\left(1-\alpha_{12}^{2}\right)^{-1 / 2} \sinh \left(\frac{1}{2} \mathcal{H} \log \frac{1+\alpha_{12}}{1-\alpha_{12}}\right) \text {, } \\
& N_{2}=1+\Im C \alpha_{12} \Im C \alpha_{12}+\Im \mathcal{C} \alpha_{12} \Im C \alpha_{12} \Im C \alpha_{12} \Im C \alpha_{12}+\cdots=\left(1-\alpha_{12}^{2}\right)^{-1 / 2} \\
& \cdot \cosh \left(\frac{1}{2} \mathfrak{C} \log \frac{1+\alpha_{12}}{1-\alpha_{12}}\right) \text {, }
\end{aligned}
$$

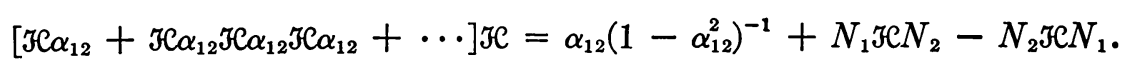

Therefore, (C3) gives

$$
\begin{aligned}
& \left\{1-\alpha_{12} \varepsilon\left(1-\alpha_{12}^{2}\right)^{-1}\right\} B_{22}=N_{2}+N_{1} \mathfrak{H C N} N_{2} \varepsilon B_{22}-N_{2} \mathfrak{H} N_{1} \varepsilon B_{22}, \\
& \left\{1-\alpha_{12} \varepsilon\left(1-\alpha_{12}^{2}\right)^{-1}\right\} B_{12}=N_{1}+N_{1} \mathfrak{H C N} N_{2} \varepsilon B_{12}-N_{2} \mathfrak{H C N} N_{1} \varepsilon B_{12} .
\end{aligned}
$$

Define

$$
M=\mathfrak{F} \tanh \left(\frac{1}{2} \mathfrak{H C} \log \frac{1+\alpha_{12}}{1-\alpha_{12}}\right),
$$


so that

$$
N_{1}=N_{2} \mathfrak{H C M} \text {. }
$$

The substitution of (C7) into (C6) gives that, after some rearrangement,

$$
\begin{aligned}
& \frac{1}{N_{2}}\left\{1-\alpha_{12} \varepsilon\left(1-\alpha_{12}^{2}\right)^{-1}+\varepsilon N_{, 2} M\right\} B_{22}=1+\operatorname{FCM} \operatorname{HC} N_{2} \varepsilon B_{22}, \\
& \frac{1}{N_{2}}\left\{1-\alpha_{12} \varepsilon\left(1-\alpha_{12}^{2}\right)^{-1}+\varepsilon N_{2} M\right\} B_{12}=\operatorname{FC} M+\operatorname{FCM} \mathcal{H C N} N_{2} \varepsilon B_{12} .
\end{aligned}
$$

Let $\alpha_{12}^{\prime}=M$ and $\alpha_{21}^{\prime}=\varepsilon N_{2}^{2}\left\{1-\alpha_{12} \varepsilon\left(1-\alpha_{12}^{2}\right)^{-1}+\varepsilon N_{2} M\right\}^{-1}$, then (C8) can be solved by the method of iteration with the results

$$
\begin{aligned}
& \frac{1}{N_{2}}\left\{1-\alpha_{12} \varepsilon\left(1-\alpha_{12}^{2}\right)^{-1}+\varepsilon N_{2} M\right\} B_{22} \\
& =1+\mathcal{H} \alpha_{12}^{\prime} \mathfrak{H C} \alpha_{21}^{\prime}+\mathcal{H} \mathcal{C} \alpha_{12}^{\prime} \mathcal{H} \mathcal{C} \alpha_{21}^{\prime} \mathfrak{H} \mathcal{C} \alpha_{12}^{\prime} \mathfrak{H} \mathcal{C} \alpha_{21}^{\prime}+\cdots, \\
& \frac{1}{N_{2}}\left\{1-\alpha_{12} \varepsilon\left(1-\alpha_{12}^{2}\right)^{-1}+\varepsilon N_{2} M\right\} B_{12}=\mathcal{F} \mathcal{C} \alpha_{12}^{\prime}+\mathcal{H} \mathcal{C} \alpha_{12}^{\prime} \mathcal{H} \mathcal{C} \alpha_{21}^{\prime} \mathcal{H} C \alpha_{12}^{\prime}+\cdots
\end{aligned}
$$

As a special case, if $\alpha_{12}=\mu^{2} \alpha_{21}$, then $B_{22}$ and $B_{12}$ can be written in closed form, and hence the series on the right hand sides of (C9) are summed even though $\alpha_{12}^{\prime} \neq$ const. $\alpha_{21}^{\prime}$. In other words, a new set of two coupled Wiener-Hopf equations with $\alpha$ replaced by $\alpha^{\prime}$ has been generated and its solution is expressible in closed form. Furthermore, this is not included in the special cases considered in Appendix B. Repeated application of the above-mentioned procedure thus produces sets of new $\alpha$ matrices, whose complexity increases rapidly.

\section{REFERENCES}

1. See, for example, B. Noble, The Wiener-Hopf technique, Pergamon Press (1958)

2. N. I. Muskhelishvili, Singular integral equations, Noordhoff Press (1953)

3. E. C. Titchmarsh, Theory of Fourier integrals, Clarendon Press (1948)

4. See p. 56 of reference 2

5. See p. 37 of reference 2 